\title{
Influência da temperatura na biologia de ninfas de Dichelops melacanthus (Dallas, 1851) (Heteroptera: pentatomidae)
}

\author{
Temperature influence on the nymphal biology of Dichelops \\ melacanthus (Dallas, 1851) (Heteroptera: pentatomidae)
}

\author{
Viviane Ribeiro Chocorosqui ${ }^{1}$; Antônio Ricardo Panizzi ${ }^{2}$
}

\section{Resumo}

Estudo referente à biologia de ninfas do percevejo barriga verde, Dichelops melacanthus (Dallas, 1851), sob três diferentes temperaturas $\left(15,20 \mathrm{e} 25^{\circ} \mathrm{C}\right)$, foi realizado em laboratório. A tentativa de testar o efeito da temperatura desde a fase de ovo foi impossibilitada devido ao fato de nenhuma ninfa eclodir de posturas a 15 e $20^{\circ} \mathrm{C}$. Ninfas do percevejo barriga verde foram mantidas sob diferentes temperaturas desde a eclosão, sendo individualizadas a partir do segundo ínstar em placas de Petri e alimentadas com vagens verdes e sementes secas de soja. A umidade relativa ( $65 \pm 5 \%)$ e a fotofase $(14 \mathrm{~h})$ foram mantidas constantes. Ninfas acondicionadas desde o $1^{\circ}$ ínstar à temperatura de $15^{\circ} \mathrm{C}$ não completaram o $2^{\circ}$ ínstar, havendo $100 \%$ de mortalidade desde o início do experimento. Sob $20^{\circ} \mathrm{C}$, somente um adulto emergiu, e a mortalidade total foi de $96,7 \%$. À temperatura de $25^{\circ} \mathrm{C}, 56 \%$ das ninfas conseguiram completar seu desenvolvimento. O tempo total de desenvolvimento foi de 56,0 dias para o único adulto (macho) obtido à $20^{\circ} \mathrm{C}$. Em $25^{\circ} \mathrm{C}$, o tempo de desenvolvimento de fêmeas foi de 24,8 dias; os machos levaram 24,0 dias para completar seu desenvolvimento. Portanto, o percevejo D. melacanthus não foi capaz de se desenvolver sob temperaturas amenas $\left(15^{\circ} \mathrm{C} \mathrm{e} 20^{\circ} \mathrm{C}\right)$ em laboratório.

Palavras-Chave: Percevejo, desenvolvimento, fator abiótico, mortalidade.

\begin{abstract}
Performane of nymphs of the green belly bug, Dichelops melacanthus (Dallas, 1851), under three different temperatures $\left(15,20 \mathrm{e} 25^{\circ} \mathrm{C}\right)$ was evaluated in the laboratory. It was impossible to evaluate the temperature effect since egg stadium, because no nymph ecloded under 15 and $20^{\circ} \mathrm{C}$. D. melacanthus nymphs were maintained under those different temperatures since the first stadium. Upon reaching the second instar, they were placed individually in Petri dishes and fed with green pods and dry seeds of soybean. The environmental cahambers were maintained with relative humidity of $65 \pm 5 \%$ and photophase of 14 hours. Nymphs maintained under $15^{\circ} \mathrm{C}$ showed $100 \%$ of mortality on the second instar. Under $20^{\circ} \mathrm{C}$, only one adult emerged; the total mortality (2nd-5th instar) was about $96,7 \%$. Under $25^{\circ} \mathrm{C}, 56 \%$ of nymphs completed
\end{abstract}

\footnotetext{
1 Engenheira Agrônoma, formada na Universidade Estadual de Londrina (dez/1995), com Mestrado em Agronomia na mesma universidade, em convênio com a Universidade de Gent, Bélgica (1996-1998), Doutorado em Entomologia na Universidade Federal do Paraná, em convênio com a Embrapa Soja, em Londrina, Paraná (1998-2001). Bolsista do Projeto "Desenvolvimento de Programa de Manejo Integrado da broca-do-cafeeiro, Hypothenemus hampei (Ferrari, 1876), com a utilização do fungo entomopatogênico Beauveria bassiana", conduzido na Universidade Estadual de Londrina, financiado pelo convênio Embrapa/ Funcafé (FAGRO). Endereço para correspondência: UEL/CCA/ Depto de Agronomia, Campus Univeersitário, Caixa Postal 6001, CEP 86051-990, Londrina, Paraná - Telefone res.: (043) 3321-7071 - com.: (043) 3371-4725

$2 \mathrm{PhD}$, pesquisador da Embrapa Soja - Rod. Carlos João Strass, Acesso Orlando Amaral, s/n, Caixa Postal 231, CEP 86001-970, Distrito de Warta, Londrina, Paraná.
} 
their development, becoming adults. The single nymph (male) from $20^{\circ} \mathrm{C}$ required 56.0 days to complete development. The developmental time (2nd-5th instar) under $25^{\circ} \mathrm{C}$ was 24.8 and 24.0 days, for females and males, respectively. These results suggest that $\mathrm{D}$. melacanthus was not able to develop under mild temperatures $\left(15^{\circ} \mathrm{C} \mathrm{e} 20^{\circ} \mathrm{C}\right)$ in the laboratory.

Key words: Stinkbug, development, a biotic factor, mortality

\section{Introdução}

Além do fotoperíodo, a temperatura é um dos estímulos abióticos de maior importância na indução da diapausa em insetos. O termoperíodo, assim como o fotoperíodo, apresenta variação sazonal, podendo ser utilizado para indicar a chegada de condições adversas ao inseto (BECK, 1983; HODEK; HODKOVÁ, 1988; LEATHER; WATERS; BALE, 1993). Entretanto, o termoperíodo está sujeito a uma grande flutuação entre os anos, sendo menos confiável como indicador sazonal. Além disso, dificilmente a temperatura é considerada o único fator abiótico que controla o estado fisiológico do inseto; de um modo geral, a temperatura age modificando ou reforçando o efeito de um outro estímulo, como o fotoperíodo (LEATHER; WATERS; BALE, 1993).

A temperatura pode influenciar no desenvolvimento dos insetos, principalmente quando há temperaturas cíclicas, ou seja, quando a temperatura varia durante 24 horas por dia, como no ambiente natural (BECK 1983). O efeito na biologia do inseto também pode ser detectado com temperaturas constantes em laboratório, porém com menor precisão.

Lin, Hodson e Richards, (1954), estudando o efeito da temperatura no desenvolvimento embrionário e na viabilidade de ovos de Oncopeltus fasciatus (Dallas, 1852), observaram que nenhuma ninfa eclodiu em temperaturas constantes de $15^{\circ} \mathrm{C}$. Kariya (1960) avaliou o efeito da temperatura no desenvolvimento e na mortalidade de Nezara viridula L., 1758 e $N$. antennata Scott, 1874. Dentre as temperaturas testadas neste estudo, a temperatura favorável para o desenvolvimento de ovo a adulto variou de 20 a $30^{\circ} \mathrm{C}$ em $N$. viridula, e 22,5 a $27,5^{\circ} \mathrm{C}$ em $N$. antennata. Nas duas espécies, a mudança de coloração ocorre mais frequentemente com a queda da temperatura. A mortalidade não variou entre as tempe- raturas testadas. Sharma e Sharma (1999) estudaram a biologia de Chauliops nigrescens Distant, 1904 (Heteroptera: Lygaeidae) em diferentes temperaturas $\left(20,25\right.$ e $\left.30^{\circ} \mathrm{C}\right)$ e umidades relativas. A emergência de adultos ocorreu em todas as temperaturas, porém o tempo de desenvolvimento foi maior nas temperaturas mais baixas.

O percevejo Dichelops melacanthus (Dallas, 1851), vulgarmente conhecido como barriga- verde, tem sido citado em culturas de verão, como a soja Glycine max (L.) Merrill, desde a década de 70 (PANIZZI et al., 1977). Entretanto, ele vem causando prejuízos às culturas de outono e inverno, como o milho (safrinha) Zea mays L. e o trigo Triticum aestivum L. (PANIZZI; CHOCOROSQUI, 1999, 2000). Este fato demonstra a adaptação dessa espécie a temperaturas mais amenas.

Em amostragens realizadas durante período de geada, ninfas e adultos do percevejo barriga-verde estavam praticamente imóveis, com as pernas voltadas para cima, porém com reação ao toque. O efeito da baixa temperatura no desenvolvimento das ninfas de D. melacanthus não é conhecido.

Portanto, este trabalho teve por objetivo estudar o efeito da temperatura na biologia de ninfas de $D$. melacanthus em laboratório.

\section{Material e Métodos}

Os estudos foram conduzidos de novembro de 2000 a maio de 2001. Adultos de D. melacanthus, espécie encontrada na região de Londrina, PR, foram coletados na área experimental da Embrapa Soja. Os insetos foram separados em casais, acondicionados em caixas gerbox $(11 \times 11 \times 3,5 \mathrm{~cm}) \mathrm{e}$ alimentados com vagens e sementes secas de soja variedade Paraná, para a produção de ovos e ob- 
tenção de ninfas. As condições ambientais utilizadas durante esta fase do experimento foram: $25 \pm 1^{\circ} \mathrm{C}, 65 \pm 5 \%$ UR e $14 \mathrm{~h}$ de fotofase.

Na primeira tentativa em se obter ninfas, os ovos foram divididos em três grupos, um para cada temperatura escolhida: 15,20 e $25^{\circ} \mathrm{C}$ (temperatura padrão). Eles foram acondicionados em placas de Petri $(9,0 \times 1,5 \mathrm{~cm})$ forradas com papel filtro umedecido. Entretanto, nenhuma ninfa eclodiu sob as temperaturas de 15 e $20^{\circ} \mathrm{C}$. Portanto, três novos grupos de ovos foram preparados e mantidos a $25^{\circ} \mathrm{C}$ até a eclosão das ninfas.

Após a eclosão, cada grupo de ninfas foi transferido para câmaras climáticas com temperaturas distintas: 15,20 e $25^{\circ} \mathrm{C}$. Na mudança para o segundo ínstar, as ninfas foram individualizadas em placas de Petri $(9,0 \times 1,5 \mathrm{~cm})$ forradas com papel filtro, contendo um recipiente plástico $(2,8 \mathrm{~cm}$ de diâmetro) com algodão umedecido, num total de 30 ninfas por temperatura. As ninfas foram alimentadas com vagens verdes e sementes secas de soja.

As ninfas foram observadas diariamente, anotando-se a mudança de ínstar e a mortalidade. Quando da emergência dos adultos, estes foram sexados e pesados em balança eletrônica (Mettler Toledo PB 303). Foram calculados o tempo de duração de cada ínstar, o tempo de desenvolvimento das ninfas $\left(2^{\circ}-\right.$ $5^{\circ}$ ínstar) e a percentagem de mortalidade em cada temperatura.

Os dados, em delineamento experimental inteiramente casualizado, foram submetidos à análise de variância, sendo a comparação de médias feita utilizando-se o teste $t$ de Student (P£0,05).

\section{Resultados e Discussão}

O desempenho de ninfas de D. melacanthus foi influenciado pela temperatura. Os ovos mantidos sob 15 e $20^{\circ} \mathrm{C}$ não deram origem a ninfas, havendo a necessidade de mantê-los à $25^{\circ} \mathrm{C}$. Ninfas acondicionadas desde o $1^{\circ}$ ínstar à temperatura de $15^{\circ} \mathrm{C}$ não completaram o $2^{\circ}$ ínstar, havendo $100 \%$ de mortalidade desde o início do experimento (Figura 1). Sob $20^{\circ} \mathrm{C}$, houve alta mortalidade no $2^{\circ}$ ínstar, restando apenas $13,3 \%$ de ninfas vivas e resultando em apenas um adulto. A mortalidade total à $20^{\circ} \mathrm{C}$ foi de $96,7 \%$. Ninfas mantidas sob temperatura de $25^{\circ} \mathrm{C}$ apresentaram mortalidade relativamente baixa, em comparação às outras temperaturas. A mortalidade no $2^{\circ}$ ínstar foi de $30 \%$. Atingiram a fase adulta $56 \%$ das ninfas. No trabalho realizado por Kehat e Wyndham (1972), o percevejo Nysius vinitor Berg. (Heteroptera: Lygaeidae) apresentou $100 \%$ de mortalidade à $12^{\circ} \mathrm{C}$; sob a temperatura de $15^{\circ} \mathrm{C}$, a mortalidade variou de 82 a $91 \%$, para ovos e ninfas desse heteróptero. Entretanto, à $20^{\circ} \mathrm{C}$, somente $30 \%$ das ninfas não conseguiram completar o desenvolvimento, mostrando a adaptabilidade desse inseto a temperaturas mais baixas do que as observadas para o percevejo barriga-verde.

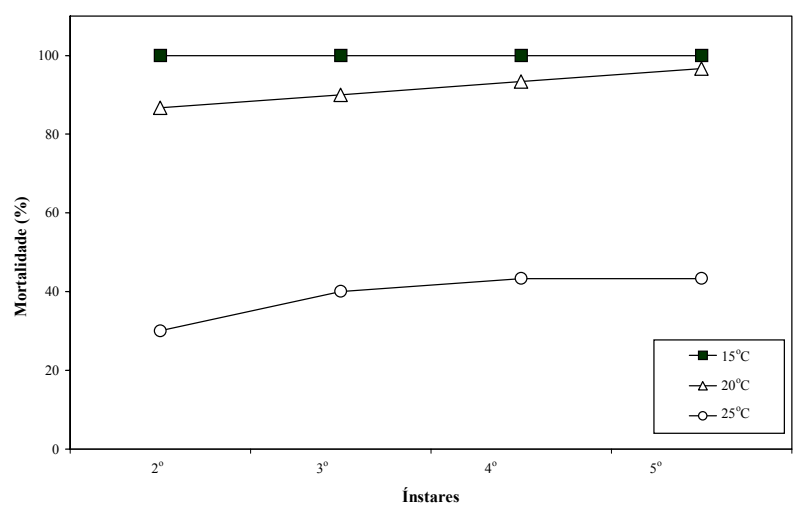

Figura 1 - Mortalidade acumulada (\%) de ninfas de Dichelops melacanthus sob diferentes condições de temperatura, alimentadas com vagens verdes e sementes secas de soja $(n=30)$.

Quanto ao tempo de desenvolvimento, houve diferença significativa entre 20 e $25^{\circ} \mathrm{C}$ Ninfas mantidas à $20^{\circ} \mathrm{C}$ levaram, em média, 12,5 dia s para completar o $2^{\circ}$ instar, enquanto as ninfas sob $5^{\circ} \mathrm{C}$ demoraram 6,4 dias, em média (Tabela 1 ). $\mathrm{No} 3^{\circ}$ e $4^{\circ}$ ínstares, ninfas provenientes da criação à $20 \mathrm{C}$ apresentaram tempo de desenvolvimento quase rês vezes maior do q ie ninfas à $25^{\circ} \mathrm{C}$. Durante o 5 ínstar, a análise esta stica não foi realizada devido ausência de repeticóes; entretanto, à $20^{\circ} \mathrm{C}$, o temp gasto para completar este ínstar foi superior ao apresentado por ninf $\mathrm{s}$ sob $25^{\circ} \mathrm{C}$. O tempo total de esenvolvimento 
foi de 56,0 dias para o único adulto (macho) obtido à $20^{\circ} \mathrm{C}$. Em $25^{\circ} \mathrm{C}$, o tempo de desenvolvimento de fêmeas foi de 24,8 dias; os machos levaram 24,0 dias para completar seu desenvolvimento. Os resultados estão de acordo com os observados por Sharma e Sharma (1999) para C. nigrescens; quanto menor foi a temperatura testada, maior o tempo de desenvolvimento em todos os estádios.

$\mathrm{O}$ peso fresco no $1^{\circ}$ dia de vida adulta foi respectivamente de 50,2 e $46,7 \mathrm{mg}$, para fêmeas e machos à $25^{\circ} \mathrm{C}$. O único macho que emergiu à $20^{\circ} \mathrm{C}$ pesou $37,0 \mathrm{mg}$.

Portanto, o percevejo D. melacanthus não se apresentou bem adaptado a temperaturas amenas constantes $\left(15^{\circ} \mathrm{C}\right.$ e $\left.20^{\circ} \mathrm{C}\right)$ em laboratório, sendo obtido apenas um adulto à $20^{\circ} \mathrm{C}$. O tempo de desenvolvimento foi maior quando as ninfas foram mantidas à temperatura de $20^{\circ} \mathrm{C}$, em comparação à $25^{\circ} \mathrm{C}$. A campo, a temperatura apresenta flutuações durante todo o dia, fato este que pode permitir o desenvolvimento de ninfas nas horas mais quentes do dia.

Tabela 1 - Tempo de desenvolvimento (dias) de ninfas e peso fresco (mg) de adultos de Dichelops melacanthus, alimentados com vagens verdes e sementes secas de soja, sob diferentes condições de temperatura (número de ninfas entre parênteses).

\begin{tabular}{|c|c|c|c|c|c|c|c|c|}
\hline \multirow{3}{*}{ Temperatura } & \multicolumn{6}{|c|}{ Tempo de desenvolvimento $^{1}$} & \multicolumn{2}{|c|}{ Peso fresco } \\
\hline & \multirow[t]{2}{*}{$2^{2}$ ínstar } & \multirow[t]{2}{*}{$3^{0}$ ínstar } & \multirow[t]{2}{*}{$4^{\circ}$ ínstar } & \multirow[t]{2}{*}{$5^{\circ}$ ínstar } & \multicolumn{2}{|c|}{$2^{-}-5^{-}$ínstar } & \multirow[b]{2}{*}{ Fêmeas } & \multirow[b]{2}{*}{ Machos } \\
\hline & & & & & Fêmeas & Machos & & \\
\hline $15^{\circ} \mathrm{C}$ & - & - & - & - & - & - & - & - \\
\hline \multirow[t]{2}{*}{$20^{\circ} \mathrm{C}$} & $12,5 \pm 1,66 \mathrm{a}$ & $13,0 \pm 1,15 \mathrm{a}$ & $14,0 \pm 1,00 \mathrm{a}$ & $20,0 \pm 0,00^{2}$ & - & $56,0 \pm 0,00^{2}$ & - & $37,0 \pm 0,00^{2}$ \\
\hline & (4) & (3) & (2) & (1) & & (1) & & (1) \\
\hline \multirow[t]{2}{*}{$25^{\circ} \mathrm{C}$} & $6,4 \pm 0,28 \mathrm{~b}$ & $5,5 \pm 0,57 \mathrm{~b}$ & $5,5+0,40 \mathrm{~b}$ & $7,8 \pm 0,30$ & $24,8 \pm 1,18$ & $24,0 \pm 0,82$ & $50,2 \pm 2,01$ & $46,7 \pm 2,78$ \\
\hline & (21) & (18) & (17) & (17) & (13) & (4) & (13) & (4) \\
\hline
\end{tabular}

${ }^{1}$ Médias $(\mathrm{X} \pm \mathrm{EP})$ seguidas pela mesma letra nas colunas não diferem significativamente entre si pelo teste $t$ de Student (P£0,05).

${ }^{2} \mathrm{~A}$ análise estatística não foi aplicada quando um dos tratamentos apresentou somente uma repetição.

\section{Referências}

BECK, S. D. Insect thermoperiodism. Annual Review of Entomology, Stanford, v.28, p.91-108, 1983.

HODEK, I.; HODKOVÁ, M. Multiple role of temperature during insect diapause: a review. Entomologia Experimentalis et Applicata, Dordrecht, v.49, p.153-165, 1988.

KARIYA, H. Effect of temperature on the development and the mortality of the southern green stink bug, Nezara viridula and the oriental green stink bug, $N$. antennata. Kontyu, Tokyo, v.14, p.242-246, 1960.

KEHAT, M.; WYNDHAM, M. The influence of temperature on development, longevity, and fecundity in the rutherglen bug, Nysius vinitor (Hemiptera: Lygaeidae). Australian Journal of Zoology, Victoria, v. 20, p. 67-78, 1972.
LEATHER, S. R.; WALTERS, K .F. A..; BALE, J. S. The ecology of insect overwintering. Cambridge University Press, 1993.

LIN, S.; HODSON, A .C.; RICHARDS, A. G. An analysis of threshold temperatures for the development of Oncopeltus and Tribolium eggs. Physiological Zoology, Chicago, v.27, p.287-311, 1954.

PANIZZI, A. R. et al. Insetos da soja no Brasil. Londrina: EMBRAPA/CNPSo, 1977. (Boletim Técnico, 1)

PANIZZI, A. R.; CHOCOROSQUI, V. R. Pragas: elas vieram com tudo. Cultivar, Pelotas, v.11, p.8-10, 1999.

. Os percevejos inimigos. A Granja, Porto Alegre, v.616, p.40-42, 2000.

SHARMA, D.; SHARMA, K. C. Biology of the black bean bug (Chauliops nigrescens) (Hemiptera: Lygaeidae) at different temperature and humidity conditions. Indian Journal of Agricultural Sciences, New Delhi, v.69, p.804$805,1999$. 